\title{
Developing a novel ice protection system for wind turbine blades using vibrations of both short and long wavelengths
}

\author{
Hossein Habibi*, Graham Edwards`, Liang Cheng", Haitao Zheng*, Adam Marks`, Vassilios Kappatos*, \\ Cem Selcuk*, Tat-Hean Gan*
}

\footnotetext{
* Brunel Innovation Centre (BIC), Brunel University, Uxbridge, Middlesex, UB8 3PH, United Kingdom ${ }^{\dagger}$ TWI Ltd, Granta Park, Great Abington, Cambridge, CB21 6AL, United Kingdom
}

\begin{abstract}
Icing conditions in cold regions of the world may cause problems for wind turbine operations, since accreted ice can reduce the efficiency of power generation and create concerns regarding ice-shedding. This paper covers modelling studies and some experimental development for an ongoing ice protection system that provides both deicing and anti-icing actions for wind turbine blades. The modelling process contained two main sections. The first part involved simulation of vibrations with very short wavelength or ultrasonic guided waves (UGW) on the blade to determine optimal excitation frequency and transducer configuration. This excitation creates horizontal shear stress at the interface between ice and blade and focuses energy at the leading edge for de-bonding ice layers. The second modelling approach simulated the effects of vibrations with very long wavelength along with estimation of fatigue life due to harmonic forces to characterise the best parameters for shaker(s) mounted on blades. In parallel with this study, an empirical array of novel resonating shear transducers has been developed using a Design of Experiments (DoE) approach to demonstrate the practicability of inducing shear horizontal waves at the leading edge of wind turbine blades. This experimental verification also makes it possible to investigate the many parameters influencing ice-removal. In addition, piezo-electric and macro-fibre composite actuators have been investigated in place of conventional electromagnetic shakers, in order to save weight and simplify integration of the deicing system components. The ongoing research is intended to provide an active solution for
\end{abstract}

icing prevention and deicing, enabling safe and reliable operation of wind turbines in adverse weather conditions.

Keywords: Wind turbine blades, deicing, ultrasonic guided waves, low frequency vibration, fatigue

\section{Introduction and developed approach}

Today, many of the windy areas suitable for the harvest of wind energy are in severely cold places which expose wind turbines to the danger of icing. Ice accretion on turbines blades could lead to significant reduction in their efficiency through unfavourable changes in the aerodynamic performance of the blade profile. Icing also has other impacts on wind turbines including accelerated wear in structural components and the risk of damage from ice thrown from blades: an especial problem when turbines are installed close to residential areas.

The available techniques for tackling ice formation on blades have limitations for full industrial use. For example, chemicals sprayed on blades do not remain on their surfaces for a long time and applied coatings to surfaces cannot successfully prevent ice formation [1]. Likewise all thermal deicing techniques consume large amounts of generated power: the power needed for ice protection can consume up to $12 \%$ and $15 \%$ of the turbine's nominal power output in the cases of electrical resistance heating and hot air circulation respectively [2]. Another disadvantage of thermal techniques is the risk of composite delamination due to excessive 
temperature if they are not protected by sensors and appropriate control systems. The current negative effects of available methods call for development of an alternative safe, reliable and efficient ice removal technology.

The current development, uses ultrasonic guided waves which make the structure vibrate with very short wavelength. Ultrasonic guided waves have been widely used for non-destructive testing, with waves typically propagating at between $20 \mathrm{kHz}$ and $100 \mathrm{kHz}$ for long-range ultrasonic inspection. However this method is relatively new for large scale surface cleaning or deposit removal. Venna et al [3], for example, applied ultrasound waves with the frequency $\mathrm{f}=1 \mathrm{kHz}$ on an Aluminium airfoil structure to find and match right resonance frequencies for deicing the airfoil. Likewise JL Palacios [4] tested ultrasound waves for helicopter blade anti-icing and deicing using two distinct modes; transverse and shear being effective in both short ranges and longer distances. Power consumption recorded in this study was up to $0.37 \mathrm{~W} / \mathrm{cm}^{2}$ which is low compared to the electro-thermal techniques which are reported to consume around 3.8 $\mathrm{W} / \mathrm{cm}^{2}$ under similar conditions [4]. Part of the modelling studies of the current research has been built on the work done by [4]. In this context, Overmeyer et al., [5], have also implemented UGW for structures representative of rotorcraft blade leading edges through a new de-icing bondline design for increasing ice interface transverse shear stresses. It is notable that despite fair improvement of UGW performance (compared to similar work in [4]), the power consumption of the de-icing system was still low at an average of $0.63 \mathrm{~W} / \mathrm{cm}^{2}$.

Low frequency vibration as an ice protection system is a joint method in this work which was first used by Bell Helicopter in 1978 as a feasibility study on helicopter blades [6]. This experimental setup was based on vibrating helicopter blades in flexural bending or torsional modes. The appropriate frequencies had to be close to the blade's major resonance frequencies for generating maximum acceleration at its surface to shake off the ice. According to the results of this research, a total acceleration of 25 to $30 \mathrm{~g}$ obtained at the first 6 to 8 mode shapes of the structure could cause effective removal of accreted ice. Nevertheless it was found that this sort of vibration, although effective across most of the blade surface was somewhat inefficient at the leading edge which is the most critical area for ice accretion (see Fig. 1 for position of the leading edge). For this reason, the current study has combined low frequency vibrations (with very long wavelength) and ultrasonic guided waves (with very short wavelength) to compensate for the shortcomings of each approach. So this approach considers introduction of energy into the blade structure by two types of vibrations. Low frequency vibrations induce total acceleration to the blade surface, while UGWs create shear stress on the leading edge of the blade. In combination, there are intended to either weaken the ice-blade interface or instantly shed the ice layer. Part of the current work has been devoted to computer simulation which plays a significant role in verifying the ability of waves to propagate in a composite blade. Modelling results also allow determination of the best potential locations and characteristics of dynamic forces for shakers to generate required accelerations in the critical areas of the blade without posing a risk of fatigue damage.

To find natural frequencies and mode shapes of the blade for vibration, Finite Element Methods (FEM) based on approximate numerical solutions of Partial Differential Equations (PDEs) were used via ANSYS software. The geometrical surfaces of the blade were also simulated in this software (see Fig.1) with different thicknesses of $0.007 \mathrm{~m}, 0.009 \mathrm{~m}$ and 0.0156 $\mathrm{m}$ for skin, spar (shear webs) and caps of the blade respectively. 


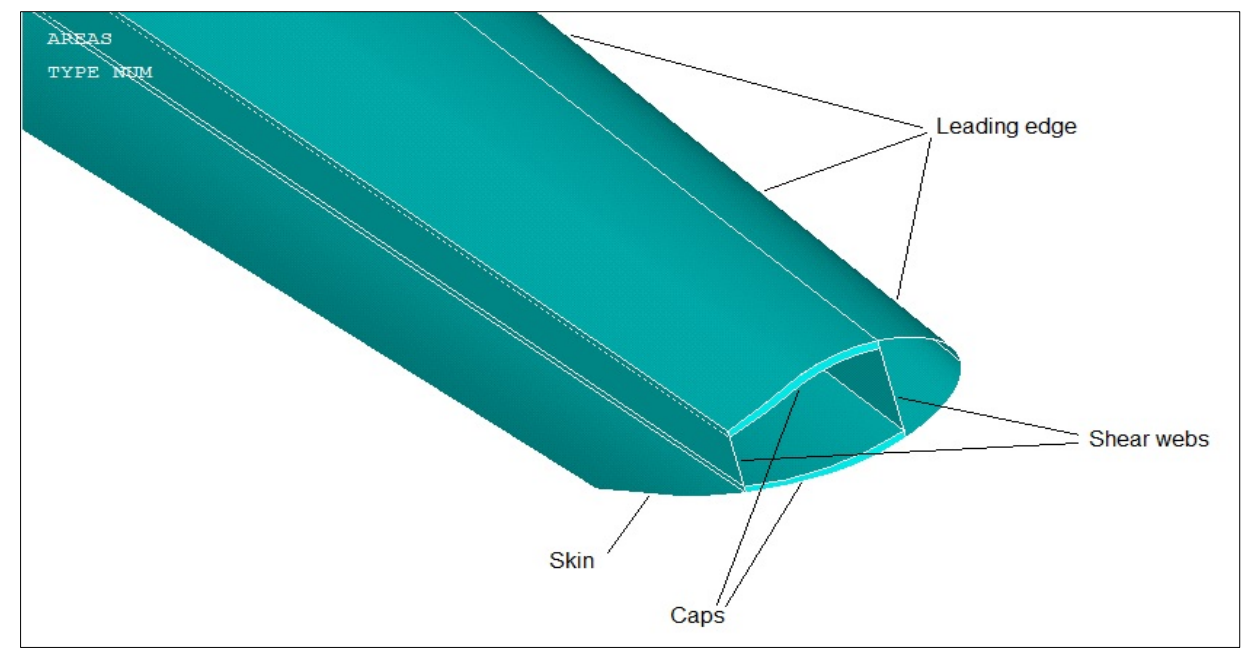

Fig. 1: A cut, 3D view of the wind turbine blade close to its tip modelled in ANSYS which clarifies some features.

The wind turbine model studied here is a $7.5 \mathrm{~m}$ composite blade from a $75 \mathrm{~kW}$ turbine using basic NACA 44xx airfoil series [7], [8]. Table 1 shows mechanical properties of orthotropic glass-reinforced composite from which the blade is built. The density of this composite material is $\rho=1860 \mathrm{~kg} / \mathrm{m}^{3}$ and its damping coefficient has been considered as $\zeta$ $=0.003[9]$.

\section{Simulation and modelling results}

\subsection{Ultrasonic guided waves}

The idea of UGW which was initially used by Palacios et al $[4,10]$ for deicing helicopter blades is based on generating high enough shear stress at the ice-blade interface to exceed the ice adhesion strength. An important concept developed in other surveys on deicing by UGW, is called Interfacial Stress Concentration Coefficients (ISCC) to calculate a normalized interface shear stress [11].

ISCC is defined by the following equation when the axis $x$ is the direction of wave propagation:

$$
I S C C=\frac{\left.\sigma_{y z}\right|_{\text {layer interface }}}{\sqrt{\text { power }}}
$$

where $\sigma_{y z}$ is the stress tensor in the yz plane at the interface between sample and ice, and power is defined by the following equation where $z$ is the direction along thickness,

$$
\text { power }=\int_{\text {thickness }}^{0} P_{x} d z
$$

$P$ is the Poynting's vector defining the power flow on the structure and $\mathrm{P}=\left[P_{x}, P_{y}, P_{z}\right]$. The parameter $P$ governs the equation below:

$$
P=\frac{-\vec{v}^{*} \cdot \sigma}{2}
$$

where $\vec{v}$ is particle velocity, $*$ is the complex conjugate and $\sigma$ is the stress tensor. This quantity is used to optimize frequency, mode and power needed in maximising interface shear stress for a given power. In fact, dispersion curves for the blade superimposed with ISCC values determine the best frequency and wave mode at which the blade must be excited by ultrasound transducers.

Table 1: Mechanical properties of fiberglass composite used in the blade model

\begin{tabular}{|c|c|c|c|c|c|c|c|c|c|}
\hline $\begin{array}{c}\text { Mechanical } \\
\text { properties }\end{array}$ & $\begin{array}{c}E_{1} \\
(\mathrm{GPa})\end{array}$ & $\begin{array}{c}E_{2} \\
(\mathrm{GPa})\end{array}$ & $\begin{array}{c}E_{3} \\
(\mathrm{GPa})\end{array}$ & $\begin{array}{c}G_{12} \\
(\mathrm{GPa})\end{array}$ & $\begin{array}{c}G_{13} \\
(\mathrm{GPa})\end{array}$ & $\begin{array}{c}G_{23} \\
(\mathrm{GPa})\end{array}$ & $v_{23}$ & $v_{12}$ & $v_{13}$ \\
\hline $\begin{array}{c}\text { STEF-1 glass } \\
\text { fabric }\end{array}$ & 5.62 & 4.59 & 4.59 & 0.406 & 0.406 & 0.28 & 0.24 & 0.22 & 0.22 \\
\hline
\end{tabular}




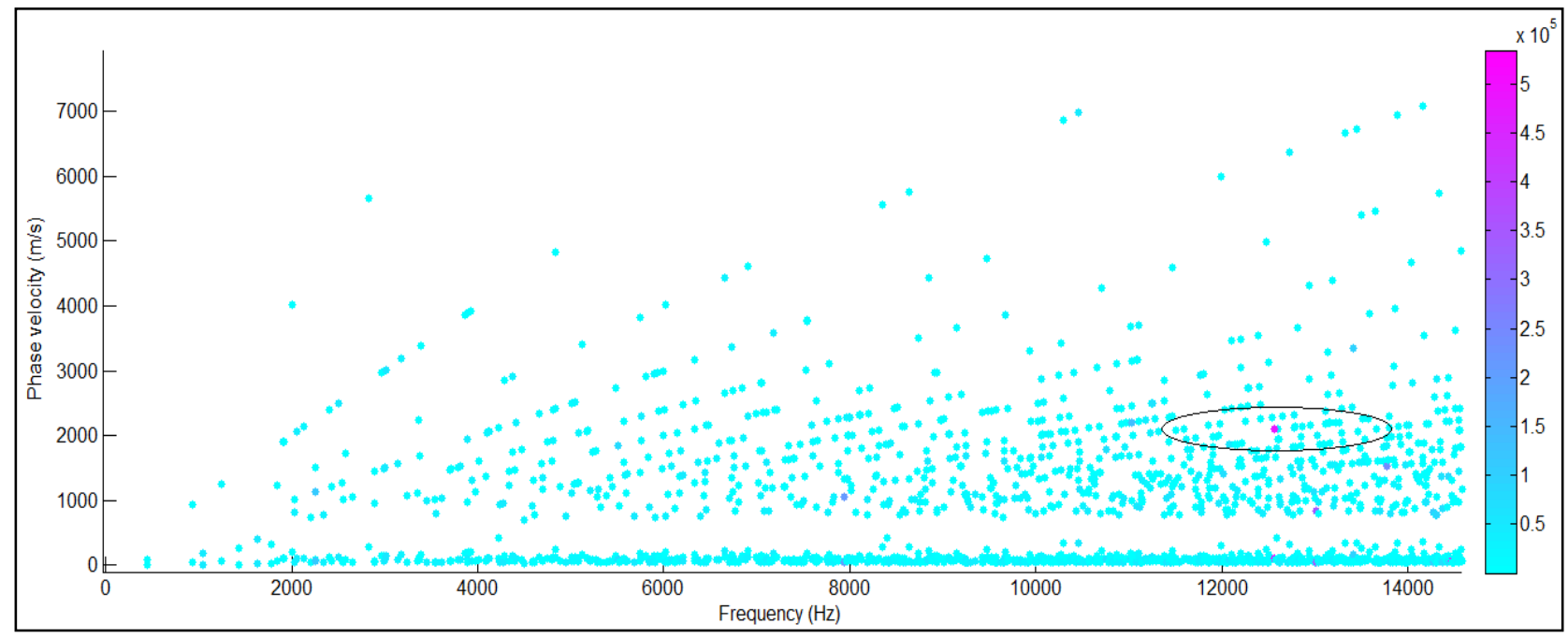

Fig. 2: Combination of dispersion curve and ISCC curves for the blade with $2 \mathrm{~mm}$-thick glaze ice to find non-dispersive modes of high ISCC such as circled area

The criteria for selecting the central frequency and wave mode for ultrasonic deicing are then: $a$ ) The larger the ISCC, the less power would be required for the de-icing system to be effective, $b$ ) the central frequency and wave mode should be nondispersive in dispersion curves. As an example, Fig. 2 represents dispersion curves superimposed with ISCC results for a $7 \mathrm{~mm}$ thick leading edge composite blade covered by a $2 \mathrm{~mm}$ ice layer. Based on these results, optimal central frequency, phase velocity and wave length of excitation signal should be chosen as $12.56 \mathrm{kHz}, 2093.5 \mathrm{~m} / \mathrm{s}, 0.1667 \mathrm{~m}$ respectively. These are the values to be used for focusing UGW power via a transducer arrangement. For this reason a sinusoidal signal was applied for the transducer to excite waves in the blade at $\mathrm{f}=12.56 \mathrm{kHz}$ as the central frequency. A pair of transducers was utilised with a gap of $\lambda / 4$ in the leading edge as shown in Fig. 3. This distance enhanced the constructive and overlapping effects of the waves launched by one another. The distribution of von Mises stress upon the blade at three different times is shown in Fig. 4. It is clear that the ultrasonic wave can be guided and its power concentrated on the leading edge, subsequently dissipating towards the areas further from the blade centre. As seen in Fig. 4, the stress created at the blade surface is above $0.5 \mathrm{MPa}$ which matches the required stress reported in the relevant literature for ice removal (see [1214]). Also the power consumed for deicing performance within this short time domain $(0.001 \mathrm{sec})$ worked out as $100 \mathrm{~W}$ for each transducer. Considering 4 pairs of transducers to cover the entire length of a blade, each blade needs $800 \mathrm{~W}$ which leads to a total consumption of $2400 \mathrm{~W}$ for a 3blade wind turbine. This is only $3.2 \%$ of a 75 $\mathrm{kW}$ wind turbine's nominal power which is low compared to the power consumption of the thermal deicing techniques [2].

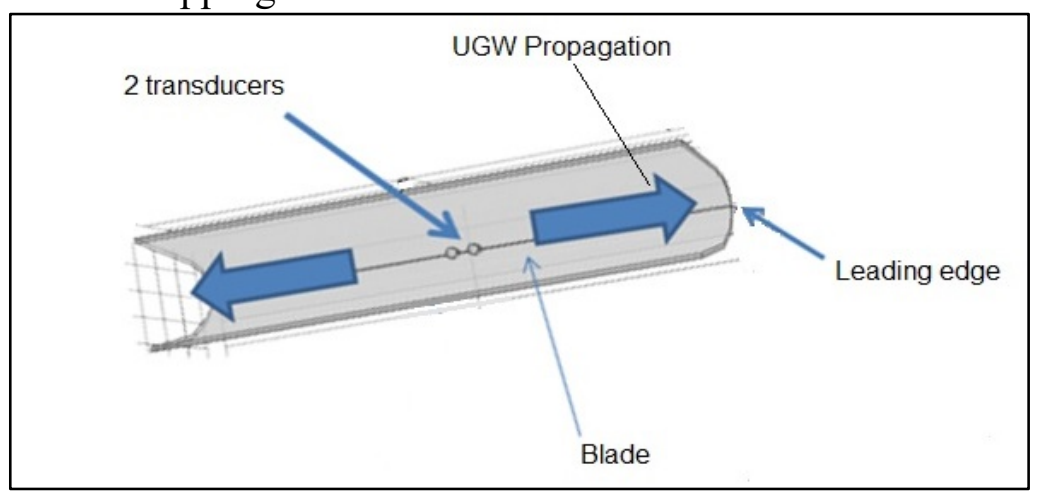

Fig. 3: Configuration of two transducers on the leading edge of the blade 


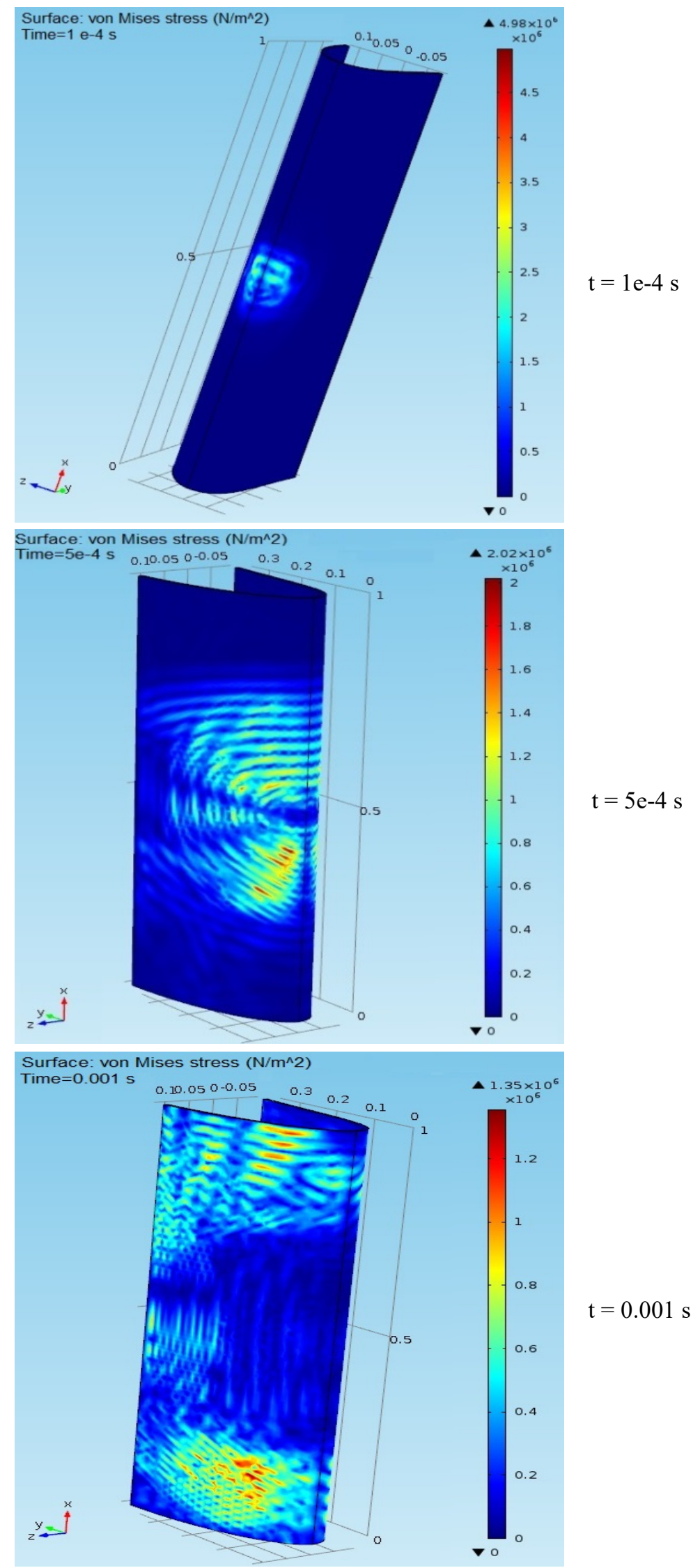

Fig. 4: Distribution of von Mises stress upon the leading edge of the composite blade over time with 2$\mathrm{mm}$ thick ice showing wave propagation and coverage areas across $1 \mathrm{~m}$ of the leading edge from $\mathrm{t}=100 \mu \mathrm{s}$ to $\mathrm{t}=1 \mathrm{~ms}$ 


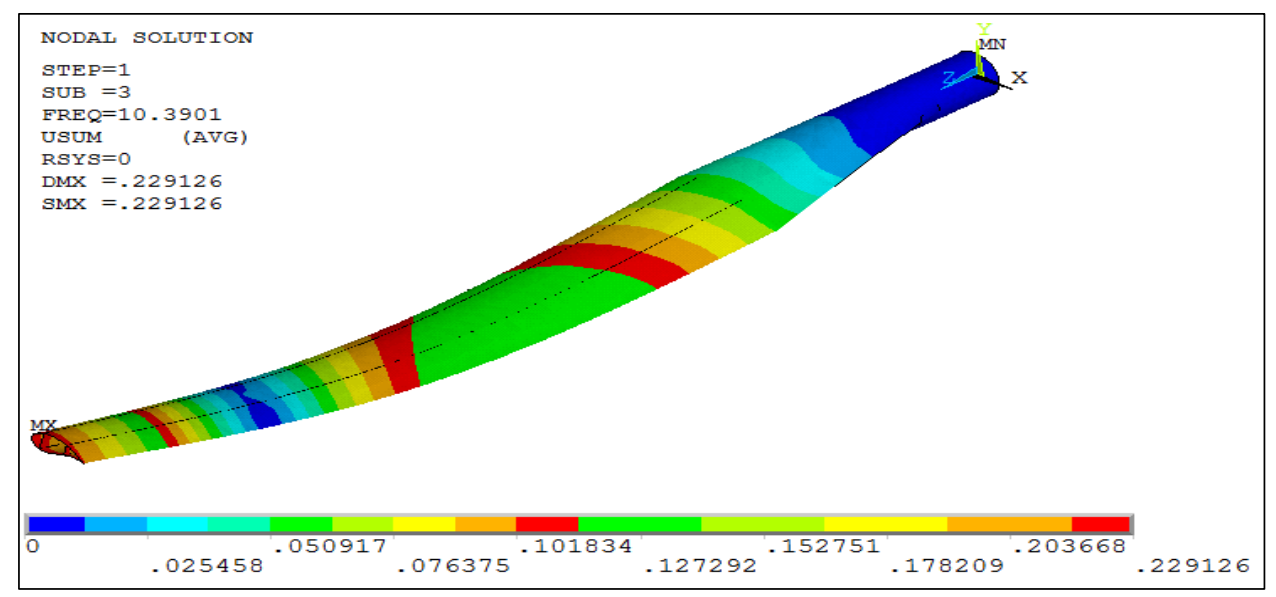

Fig. 5: The third mode shape (second flexural bending mode in flapwise direction) of the developed blade model

\subsection{Low-frequency vibrations and fatigue life estimation}

Vibrations of long wavelength or low frequency are aimed at applying the largest possible acceleration onto the turbine blade surface for shaking off ice while avoiding any damage risk. To achieve this aim, the blade needs to be excited at a frequency close to one of its low resonance frequencies. Modal and harmonic analyses may then be used to define an optimum topology as well as the best dynamic forces for vibrators. To avoid potential hazardous effects due to these harmonic forces, a fatigue analysis has to be considered as well. In the current work, an initial modal analysis was accomplished using the FEM model to obtain mode shapes and dynamical behaviour for the blade. To solve the eigenvalue problem of this system, the Block Lanczos Method was used through a Lanczos algorithm.
The first three natural frequencies of the blade which are usually predominant modes in frequency response of mechanical structures were calculated as 2.2, 3.81 and $10.39 \mathrm{~Hz}$. The third mode shape (i.e., the second flexural bending mode in flapwise direction), for example, is shown in Fig. 5 while distribution of total displacement is also displayed upon the blade surface.

Modal analysis also underlies harmonic analysis to find the best locations for installing vibrators. This depends on kinematic factors such as superposition of mode shapes, maximum displacements and nodal points in each mode shape. Evaluating the dynamic response of the blade in each mode shape based on these factors, the three points 1, 2 and 3 marked in Fig. 6 were determined as the best sites for a shaker array in both directions $x$ and $y$.

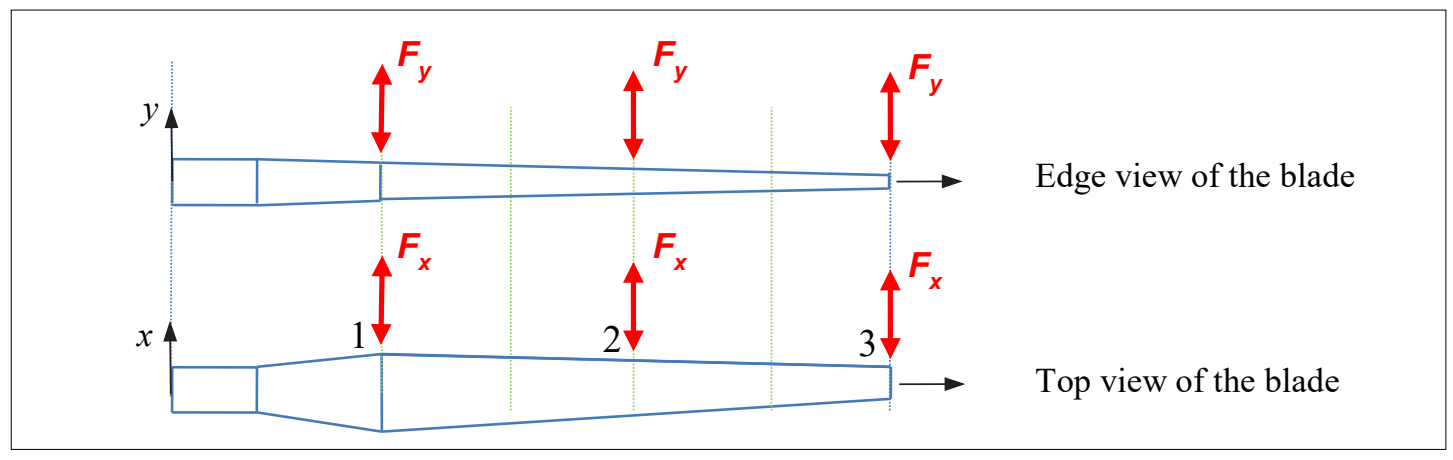

Fig. 6: Potential points for applying vibrator forces on the wind turbine blade 


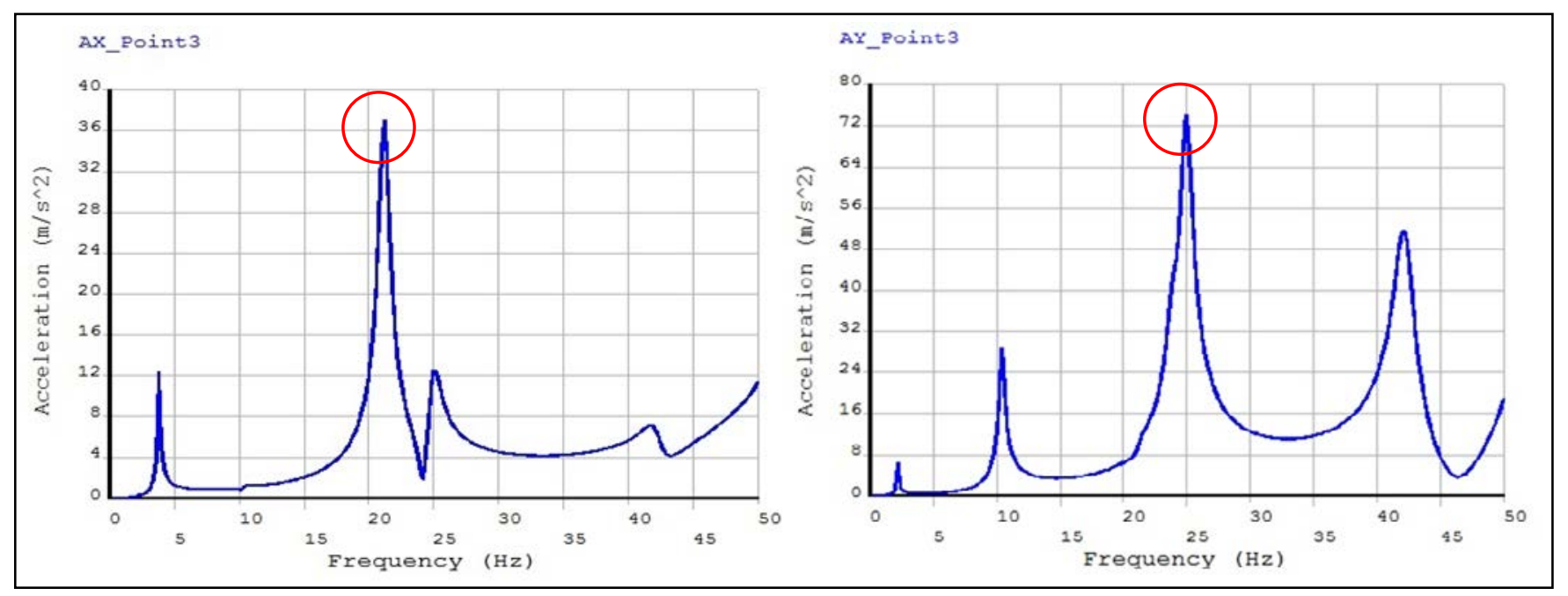

Fig. 7: Acceleration calculated for the blade tip in the frequency range [0 50] Hz;a) Edgewise direction $b$ ) Flapwise direction.

The harmonic analysis presumed that the applied loads and the steady-state response varied sinusoidally (harmonically) with time. The general equation of motion of a system subjected to external harmonic force is presented as a complex equation of harmonic motion. In this work, harmonic analysis was carried out over the frequency range [0 - 50] Hz. Two 100-N harmonic forces as $F_{\mathrm{x}}$ in direction $x$ and $F_{\mathrm{y}}$ in direction $y$, to excite modes in both flapwise and edgewise directions, were applied to the blade at a same time. The response then includes all flexural bending and torsional modes.

A survey was carried out to identify the frequencies at which total acceleration was maximised, which subsequently led to characterisation of the maximum acceleration for two different types of modes, i.e., Flapwise and Edgewise. The acceleration curves for the tip of blade which experienced highest oscillations in both directions $\mathrm{x}$ and $\mathrm{y}$ are shown in Fig.7 versus excitation frequency. As indicated in Fig.7(a), maximum acceleration emerged in the fourth mode shape or the second flexural bending mode in the edgewise direction at $\mathrm{f}=21.18$ Hz. Accordingly, Fig.7(b) suggests that maximum acceleration in the flapwise direction occurred at $\mathrm{f}=24.89 \mathrm{~Hz}$ which is the natural frequency of the sixth mode shape or the first torsional mode. So these two frequencies $(21.18 \& 24.89 \mathrm{~Hz})$ are the optimum ones at which required acceleration for deicing performance could be achieved using harmonic loading by shakers.

It worth mentioning that peak values of stress and total displacement as unfavourable parameters unlike acceleration happen at the lowest mode shape, i.e., the first flexural bending mode in the flapwise direction at $\mathrm{f}=2.2 \mathrm{~Hz}$. This means that suitable operational frequencies $(\mathrm{f}=21.18 \& \mathrm{f}=24.89$ $\mathrm{Hz}$ ) are quite different to critical mode shape. In other words the fourth and sixth modes as operational frequencies are less likely to impose failure risk due to a typical cyclic load. This is because they cause only small stresses and displacements. For this reason, reliable estimation of fatigue life would be ideally based on the first mode shape although the operational frequency for deicing is the higher mode, i.e., the first torsional mode at $24.89 \mathrm{~Hz}$.

The peak values of displacements and acceleration emerging in three points of the blade (1, 2 and 3 as marked in Fig. 6) due to applying 100-N harmonic forces in both directions $\mathrm{x}$ and $\mathrm{y}$, simultaneously on all the three potential points of the blade at the optimal frequency $(24.89 \mathrm{~Hz})$ are summarised in Table 2. The acceleration is more than $25 \mathrm{~g}$ at the tip which meets the criterion for deicing action according to the corresponding literature [6]. 
Table 2: Displacement and acceleration induced on the blade at $\mathrm{f}=24.89 \mathrm{~Hz}$ when all three points are excited simultaneously

\begin{tabular}{|c|c|c|c|c|}
\cline { 2 - 5 } \multicolumn{1}{c|}{} & \multicolumn{2}{c|}{ Displacement $(\mathrm{m})$} & \multicolumn{2}{c|}{ Acceleration $\left(\mathrm{m} / \mathrm{s}^{2}\right)$} \\
\hline & $\begin{array}{c}\text { Edgewise direction } \\
\text { (X-axis) }\end{array}$ & $\begin{array}{c}\text { Flapwise direction } \\
\text { (Y-axis) }\end{array}$ & $\begin{array}{c}\text { Edgewise direction } \\
\text { (X-axis) }\end{array}$ & $\begin{array}{c}\text { Flapwise direction } \\
\text { (Y-axis) }\end{array}$ \\
\hline Point 1 & 0.0347 & 0.0464 & 81.615 & 218.685 \\
\hline Point 2 & 0.1257 & 0.3305 & 121.642 & 252.966 \\
\hline Point 3 & 0.2590 & 0.8466 & 197.643 & 298.261 \\
\hline
\end{tabular}

Regarding points on the blade other than the tip, it is inferred that while the vibration induced at point 1 could degrade the interfacial ice bond, the entire deicing operation should be fulfilled by ultrasonic transducers. According to the US wave results, full protection against ice could be achieved by guided waves alone by mounting one pair of transducers per meter of the blade. However combining ultrasonics with the weakening effects of long wavelength vibrations would allow much easier and quicker completion of the deicing task. In terms of fatigue effects due to harmonic forces, as mentioned above, the first mode shape is the critical frequency in which maximum stress and distortion are imposed on the blade structure. Normally the areas in touch with the metal part of the blade at its root or those with high stress concentration are the most critical points of the structure on which calculations must be based (see Fig. 8, for example).

The current work has adopted a fatigue approach developed recently by Movaghghar and Lvov [8], who proposed an energy-based method to estimate fatigue life in a composite wind turbine blade. This approach which is readily applicable to different systems, only needs maximum stress appearing in the blade structure regardless the type of dynamic loading e.g. whether it is due to periodic wind pressure or transient harmonic forces. Full details on the approach conditions and its development can be found in reference [8]. The stress components $\sigma_{1}, \sigma_{2}$ and $\tau_{12}$ are the only variables needed to estimate fatigue life which were specified via ANSYS for the blade model.

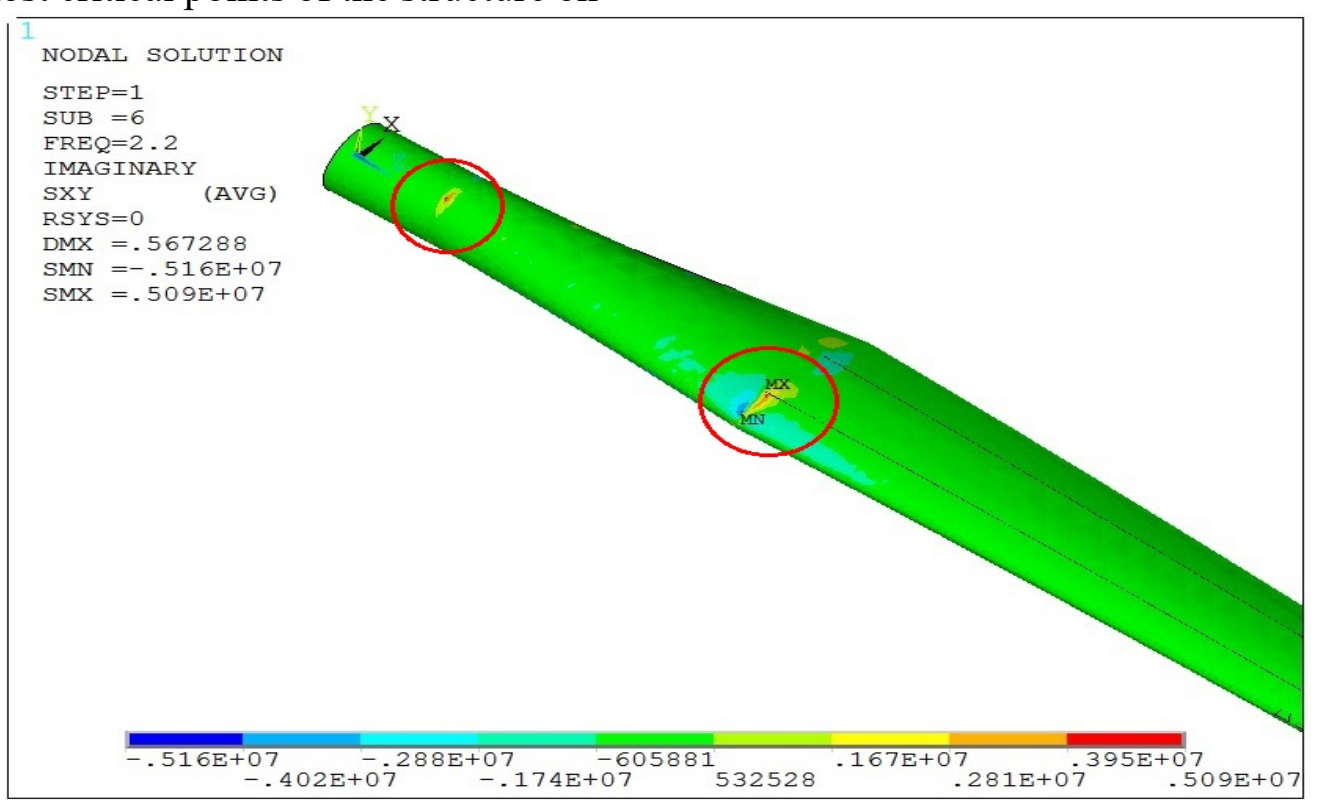

Fig. 8: Distribution of von Mises stress upon blade when $f=2.2 \mathrm{~Hz}$, due to a $100-\mathrm{N}$ harmonic force applied at the blade tip. Critical pointes are circled. 
Table 3: summary of predicted fatigue life due to applying shakers of different positions (arrays) on the blade at its first natural frequency

\begin{tabular}{|c|c|c|c|c|c|c|c|}
\hline $\begin{array}{c}\text { Shaker(s) } \\
\text { arrangement }\end{array}$ & $\begin{array}{c}\text { Single } \\
\text { Point 1 }\end{array}$ & $\begin{array}{c}\text { Single } \\
\text { Point 2 }\end{array}$ & $\begin{array}{c}\text { Single } \\
\text { Point 3 }\end{array}$ & $\begin{array}{c}\text { Dual points } \\
1 \& 2\end{array}$ & $\begin{array}{c}\text { Dual points } \\
1 \& 3\end{array}$ & $\begin{array}{c}\text { Dual points } \\
2 \& 3\end{array}$ & $\begin{array}{c}\text { All points } \\
1 \& 2 \& 3\end{array}$ \\
\hline$N_{f}$ & $2.66 \mathrm{e} 15$ & $6.36 \mathrm{e} 9$ & $1.42 \mathrm{e} 7$ & $2.61 \mathrm{e} 9$ & $1.001 \mathrm{e} 7$ & $1.54 \mathrm{e} 6$ & $1.20 \mathrm{e} 6$ \\
\hline
\end{tabular}

A normal range of what is called infinite life for GFRP material varies from $10^{5}$ to $10^{8}$ depending on the geometry of reinforcing fibres, lay-up configuration, laminate orientation, etc [15]. Table 3 presents a summary of fatigue life estimations in different loading scenarios or shaker arrays. The data given in Table 3 indicate that most of the fatigue life cycles are acceptable. Bearing in mind that shakers are supposed to work only for limited time periods during severe weather conditions, the real fatigue life is expected to be higher than calculated here due to possible stress relief between icing episodes. Moreover it should be noted that the fatigue analysis performed here due to the approach conditions was based on the critical mode shape of the blade, i.e., the first flapwise mode $(2.2 \mathrm{~Hz})$ in which the blade experiences the largest stresses and displacements. Therefore the blade is expected to have much higher life at the optimal operational frequency $(24.89 \mathrm{~Hz})$.

\section{Developing experimental validation}

The proposed use of short wavelength vibrations to remove ice at the leading edge of the wind-turbine blade relies on the propagation of Ultrasonic Guided Waves (UGW) around the outside surface of the curved leading edge from ultrasound actuators placed on the inside surface. The presence of multiple skins of highly ultrasound attenuative Glass Fibre Reinforced Plastic (GFRP) necessitates the use of high powered, low frequency ultrasound actuators. Originally in this work, the use of off-the-shelf High-Powered (HP) ultrasound actuators as used in ultrasonic cleaners was envisaged. These generate compression waves in a liquid bath in which the object to be cleaned is immersed. By a process called cavitation, these compression waves dislodge contamination from the object's surface. This process is fundamentally different to the deicing process proposed here, which will use the ultrasonic energy in the object itself rather than in the surrounding liquid. UGW energy will need to propagate through-wall from one surface to the other, and this will require very high energy levels.

The possibility of using off-the-shelf HP compression wave actuators placed on an acrylic wedge to propagate Lamb waves around the leading edge was at first investigated. This is the classical way of generating Lamb waves in a plate. It relies on the conversion of incident compression waves in the plastic to out-of-plane displacements in the plate. These displacements are the so-called Lamb waves, the most simple of which are $\mathrm{A}_{0}$ (asymmetric) and $\mathrm{S}_{0}$ (symmetric) waves. These form at ultrasound frequencies dependent on resonances that exist between plate thickness and ultrasound wavelength. The geometrical arrangement of Snell's Law determines the wavelengths of these waves.

The modelling work described previously showed that out-of-plane displacement of the surface may not provide the best solution for driving UGW with the required phase velocity. Instead it suggested that in-plane displacement of the surface, so-called Shear Horizontal (SH-waves), were a more effective way of generating shear forces of sufficient energy at the ice-GFRP interface to break the bonding.

A number of alternative actuators are available for generating SH-waves. The most commonly used in non-destructive testing are Electro-Magnetic Acoustic Transducers (EMATs), but these can only be used on conductive materials. Macro-fibre composite 
transducers can also generate SH-waves. Their main application is in morphing and stiffening structures and for energy harvesting. However, their $\mathrm{SH}$ mode generation is complicated by the bi-polar nature of the exciting forces. The widely used Piezo-electric ceramic transducers, such as Lead-Zirconium Titanate (PZT) are also available as shear motion transducers and these were the ones adopted for the experimental work done here.

The maximum power output from a PZT element will occur when it resonates and this will depend on its thickness. For example, a $0.5 \mathrm{~mm}$ PZT element might resonate at about $1.75 \mathrm{MHz}$ if the thickness mode of the actuator is triggered. PZT elements are necessarily thin and brittle and precautions are needed to prevent breakage. This was a persistent problem in designing PZT actuators for this work.

With EMATS, the transmission of SHwave modes of specific wavelength is determined by the coil or magnet spacing in the EMAT depending on its type. With PZT elements, transmission of specific SH-waves is accomplished by using arrays. If two PZT elements acting in unison are placed $1 \lambda$ apart in the $\mathrm{SH}$ wave propagation direction, then there will be constructive interference at their operating frequency. Similarly, if a second row of PZT elements is placed at right-angles to the first at a distance $1 \lambda$ apart, there will be constructive interference of wave fronts propagating in that direction too. However, with the $\mathrm{SH}$ wave front going in one direction, there is a corresponding Lamb wave front going at right angles to it, so the $\lambda$ in the right angle direction will be at $\lambda$ for the Lamb wave mode at the operating frequency. Lamb waves travel at about twice the velocity of SH-waves, so the distance between the rows will be about double that in the SHwave direction. In practice, PZT elements are not point sources of ultrasound and the wave propagation is complex. The arrays are therefore electronically tuned by phasing the signal voltages in the SH wave and Lamb wave directions to give maximum output.

As well as using arrays to select specific $\mathrm{SH}_{0}$ wave lengths, there is also the need to operate the PZT transducers at maximum power which occurs at resonance. In conventional compression wave HP transducers, this is done using the so-called Langevin design, in which the PZT element is sandwiched between two massive metal blocks, their combined thickness adding to half- $\lambda$ at the required resonance frequency. A similar design was adopted in the current work, the shear motion PZT elements being used in place of compression motion ones in the Langevin design. Experiments were conducted with aluminium, steel and acrylic blocks of different thicknesses clamped on either side of the PZT element, in order to change the resonance frequency. The acrylic blocks had two advantages: firstly they produced the shortest $\lambda$ and therefore the thinnest blocks to achieve resonance and, when used on GFRP substrates, better ultrasound acoustic impedance matching and therefore transfer of energy between the actuator and the GFRP substrate. Unfortunately, the shear PZT element's shear motion was found to generate a large amount of heat through friction, which fused the element to the acrylic blocks at relatively low voltage inputs.

Another way to improve power output was found by using stacks of PZT elements, though there were practical limitations in using more than two elements in one stack.

A parametric study was undertaken to evaluate the effectiveness of SH-wave transducers in creating stresses on a surface opposite to the actuator. Observation of deicing is difficult in ambient laboratory experiments, so sprinklings of iron powder, of the sort used in magnetic particle inspection were used to visualize the surface stresses that were present. 


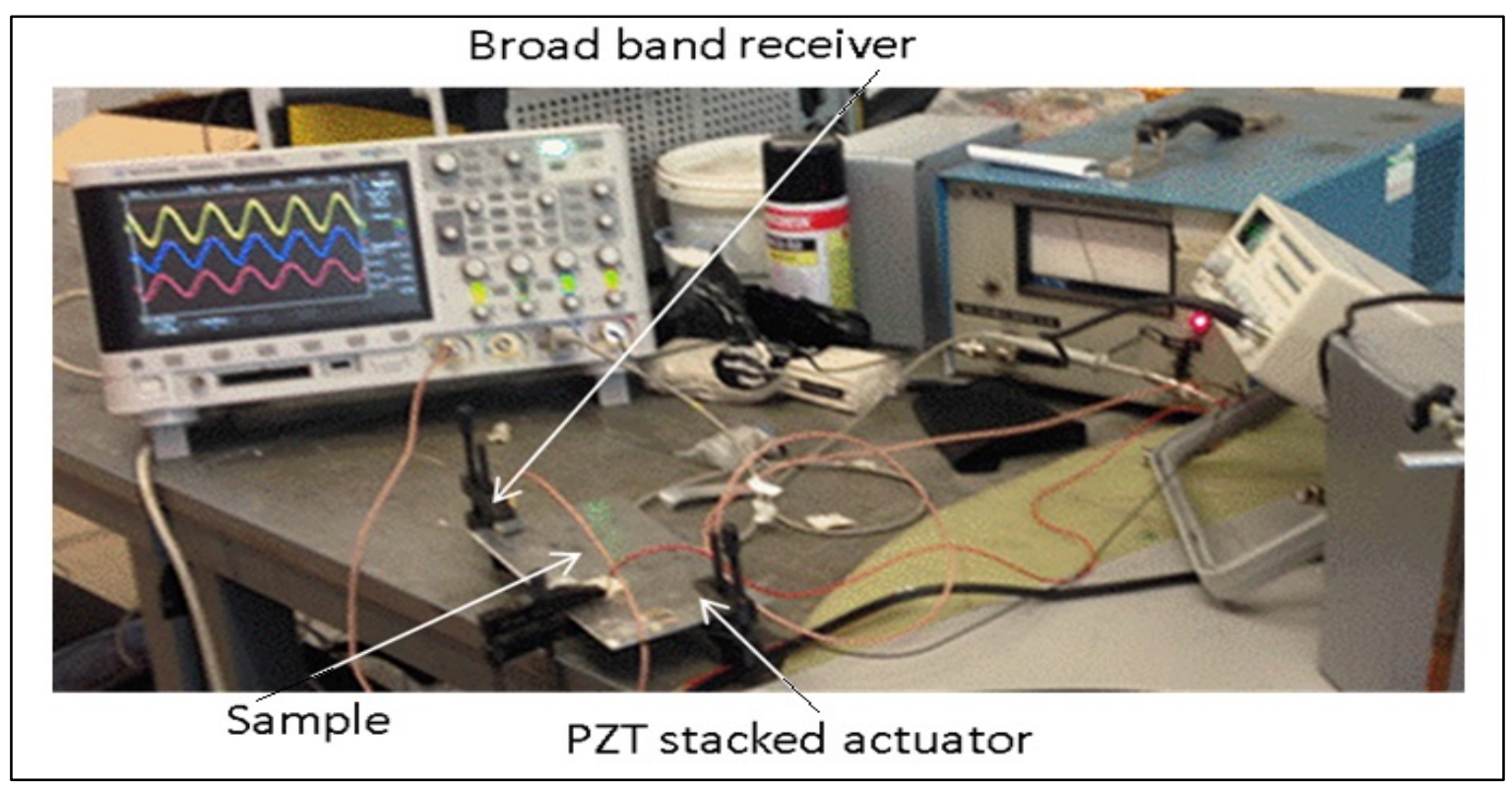

Fig. 9: Experimental set-up

A broad-band shear wave transducer, of a type used in long range ultrasonic testing to receive as well as transmit GUW was used to measure the amplitude of the $\mathrm{SH}$ waves. Frequency sweeps between $10 \mathrm{KHz}$ and $250 \mathrm{KHz}$ were controlled using a sine wave generator connected to an amplifier to raise the peak-to-peak voltages up to $200 \mathrm{~V}$. A 4channel digital oscilloscope was used to measure amplifier input and output and receiver peak-to-peak voltages and frequencies. Evaluation of these options is on-going. The experiments were conducted initially on small (50x $100 \mathrm{~mm}$ plates) in $2 \mathrm{~mm}$ thick Aluminium, $3 \mathrm{~mm}$ steel and $2 \mathrm{~mm}$ GFRP. During the frequency sweeps, magnetic particle movement occurred abruptly at certain frequencies, when patterns would appear (Fig. 10). These are patterns caused by nodes and antinodes at specific plate resonance modes. This was confirmed with numerical models. Interestingly, the particle movement did not occur at the peak voltage amplitudes from the receiver transducer. This is probably a consequence of the receiver's frequency response being affected by its own resonances.

The parameters which had the most significant effect on magnetic particle movement and $\mathrm{SH}$-wave receiver voltage were the transducer clamping force, actuator distance from the plate edges and plate clamping positions. Also, the use of Aluminium resonance blocks with PZT actuators significantly improved results. However it was found that the GFRP required such high input energy levels to cause resonance, that actuator over-heating became a problem.

The experiments continued using ice in place of magnetic particles, $1 \mathrm{~mm}$ thick patches of ice were formed on the plates held vertically in a vice at varying distances from the actuator. The time it took for the patch to become dislodged from the plate was measured. The effectiveness of different shear modes of plate resonance in removing ice is currently being investigated. 


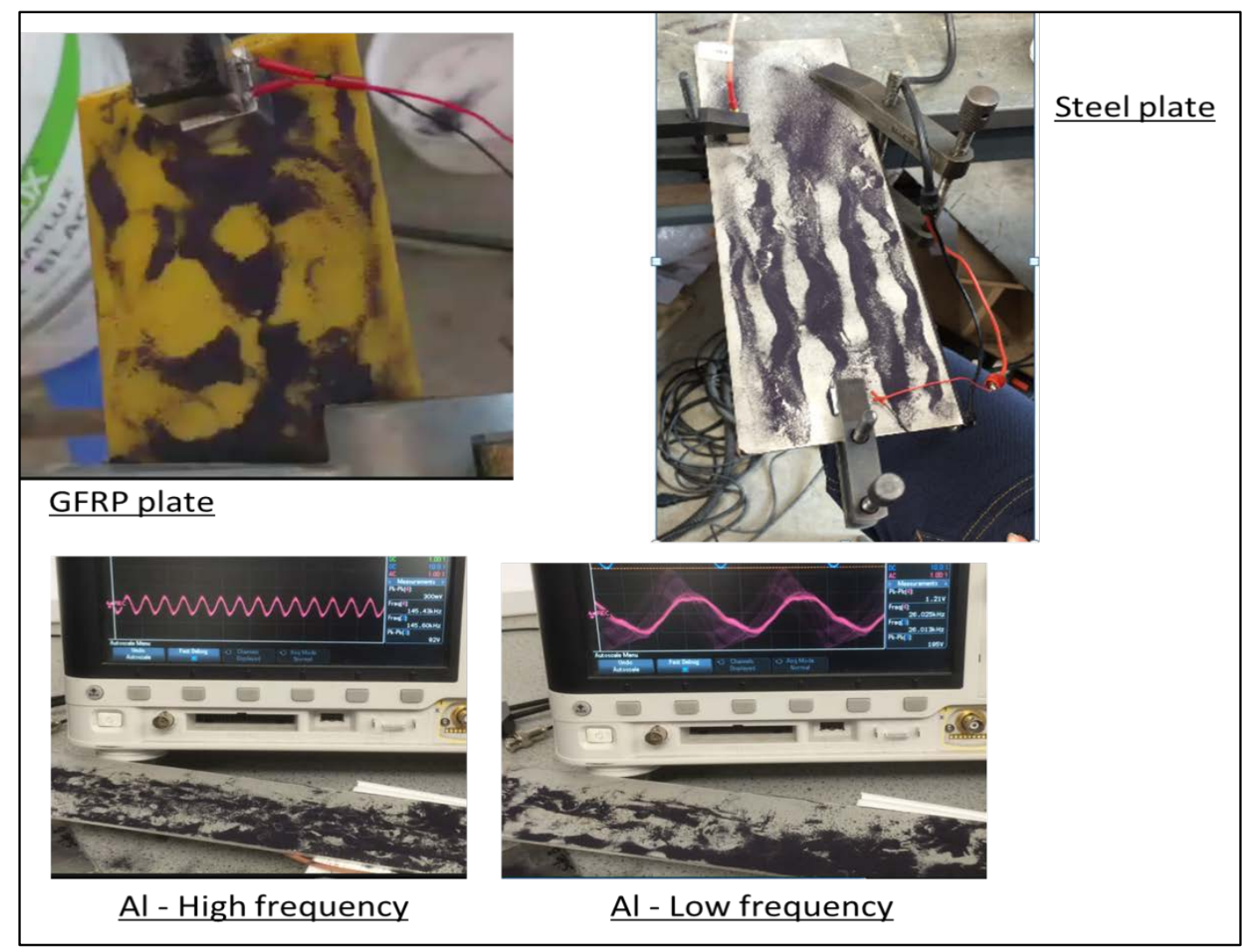

Fig. 10: SH-wave resonance patterns

With regard to evaluation of the numerical models of shaker performance, a scaled mock-up of a wind turbine has been constructed (Fig. 11). Three options for shaker deployment are being compared (Fig.12).

The simplest uses a mechanical shaker in the root of the blade. The disadvantages of this option are high weights and the generation of fatigue inducing low frequency resonances during start-up and shut-down. The preferred option from the modelling work is to use three electro-mechanical shakers positioned along the leading edge of the blade at resonance nodes. The third option is the most difficult to implement, but offers the advantage of providing more efficient bending motions to the blade instead of flexing motions. For helicopter blades this is achieved using macro-fibre composite actuators wrapped around the blade, but the much greater mass of wind turbine blades may make this method impracticable.

Once the preferred options for GUW generation and shaker operation have been selected, they will be integrated with an offthe-shelf ice-detection system and trialled on a turbine blade in an environmental chamber. 


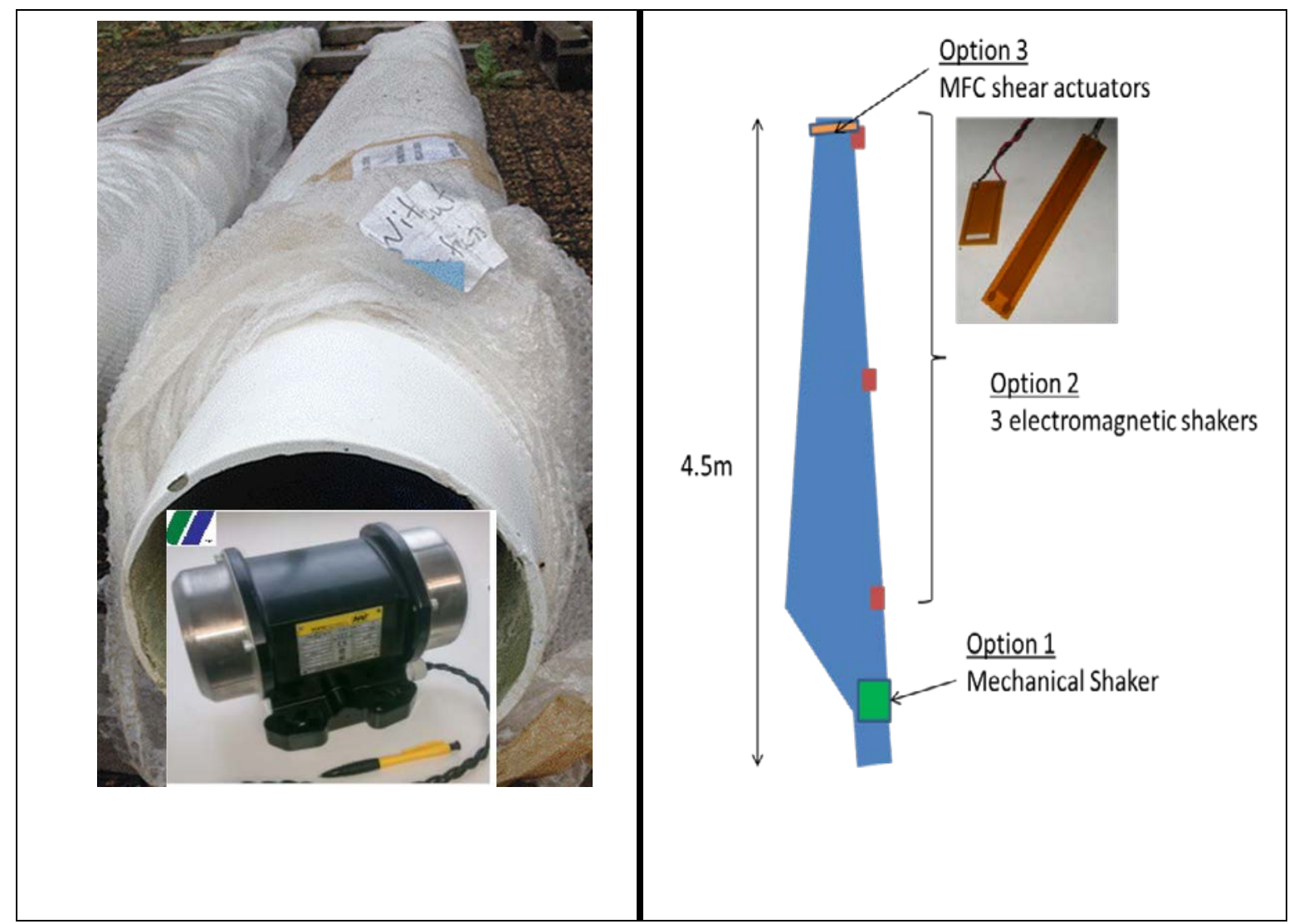

Fig. 11: Turbine blade mock-up and mechanical shaker

Fig. 12: Shaker placement options

\section{Conclusion and future work}

Modelling studies have been carried out on a proposed novel ice protection system for a typical wind turbine blade which combines two types of vibrations: very short and very long wavelength. Ongoing lab experiments and the prospective scope for full empirical validation have also been described. The current paper presents and introduction to a hybrid blade deicing method consisting of two relatively efficient approaches which compensate for each other's deficiencies. This strategy is aimed at maximising antiicing/deicing functionality with notably low power consumption (approximately $3.2 \%$ of the wind turbine nominal power) compared to available thermal techniques such as electrical resistance heating. Results show that ultrasonic waves could be appropriately guided and power concentrated along the leading edge of the blade generating high enough stress (above $0.5 \mathrm{MPa}$ ) for ice removal. In terms of low frequency vibrations, it was concluded that a threevibrator set-up could induce the required total acceleration (above $25 \mathrm{~g}$ ) on the major surface of the blade particularly the areas that cannot be covered efficiently by ultrasound waves while complying with standards of composite blades regarding fatigue life. Since each technique meets the criteria for ice removal in complementary regions, their combination will allow full coverage of the areas of interest against icing. For example, the first half of the blade (up to point 2 in Fig.6) needs to be covered by UGW transducers due to the insufficient level of acceleration caused by shakers in this area. To date, the ongoing experimental validation has shown initial compatibility with expectations obtained from modelling studies. Further experimental work will include verification of shear stress generation over the blade surface using a vibrometer to fine tune positioning of the UGW sensors. Final testing of the integrated system will involve wind tunnel trails with real icing conditions. 


\section{Acknowledgements}

The DeICE-UT project is co-founded by the European Commission through the Seventh Framework Programme (FP7/20072013) managed by REA-Research Executive Agency through the funding scheme "Research for the Benefit of SME-s" under Grant Agreement No.605138.

\section{References}

1. Kimura S., Sato T., Kosugi K., The effects of anti-icing paint on the adhesion force of ice accretion on a wind turbine blade, Proceedings of BOREAS VI International Conference, April 2004, Finish Meteorological Institute, Pyhatunturi, Finland, pp. 9.

2. Laakso T., Peltola E., Review on blade heating technology and future prospects, Proceedings of BOREAS VII International Conference, March 2005, Saariselka, Finland, pp. 12.

3. Venna S., Lin Y., Botura G., Piezoelectric Transducer Actuated Leading Edge DeIcing with Simultaneous Shear and Impulse Forces, Journal of Aircraft, Vol. 44, No. 2, 509-515, 2007.

4. Palacios J.L., Design fabrication and testing of an ultrasonic deicing system for helicopter rotor blades, PhD Thesis, the Pennsylvania State University, Engineering Science and Mechanics, 2008.

5. Overmeyer A., Palacios J., and Smith E., Ultrasonic De-Icing Bondline Design and Rotor Ice Testing, AIAA Journal, Vol. 51, No. 12 (2013), pp. 29652976. doi:10.2514/1.J052601

6. Coffman H.J., Helicopter Rotor Icing Protection Methods, Bell Helicopter Textron Inc., Fort Worth Texas, Journal of the American Helicopter Society, 1987.
7. Johnston J.F., Farone W.A. and Mikhail A., Composite Wind Turbine Rotor Blade and Method for Making Same, US Patent No. 4976587, 1990.

8. Movaghghar A. and Lvov G.I., A method of estimating wind turbine blade fatigue life and damage using continuum damage mechanics, International Journal of DAMAGE MECHANICS, Vol. 21, 81082.

9. Larwood S. and Musial W., Comprehensive Testing of Nedwind 12Meter Wind Turbine Blades at NREL, ASME Wind Energy Symposium, Reno, Nevada, January 2000.

10. Palacios J., Smith E., Rose J. L., Gao H., Ultrasonic Shear Wave Anti-Icing System for Helicopter Rotor Blades, 62nd Annual Forum Proceedings-American Helicopter Society, Phoenix, Arizona, 1492-1502, 2006.

11. Gao H. D., Rose J. L., Ice Detection and Classification on an Aircraft Wing with Ultrasonic Shear Horizontal Guided Waves, Ultrasonics, Ferroelectrics and Frequency Control, Vol. 56, Issue: 2, 334-344, 2009.

12. Ramanathan S., An Investigation on the Deicing of Helicopter Blades Using Shear horizontal Guided Waves" PhD Thesis, the Pennsylvania State University, Engineering Science and Mechanics, May 2005.

13. Brouwers E., Palacios J., Smith E.C., The Experimental Investigation of a Rotor Hover Icing Model with Shedding, Journal of America Helicopter Society, 2010.

14. Stallabrass J.R., Price R. D., On the Adhesion of Ice to Various Materials, Canadian Aeronautics and Space, 1963, 199-203.

15. Sutherland H.J., On the Fatigue Analysis of Wind Turbines, Sandia National Laboratories, Albuquerque, New Mexico 87185-0708, June 1999. 
